\title{
Highly sensitive detection of leukemia cells based on aptamer and quantum dots
}

\author{
YATING YU $^{1 *}$, SILIANG DUAN $^{1 *}$, JIAN HE $^{1 *}$, WEI LIANG ${ }^{1}$, JING SU $^{1}$, \\ JIANMENG ZHU ${ }^{1}$, NAN HU ${ }^{1}$, YONGXIANG ZHAO ${ }^{1}$ and XIAOLING LU ${ }^{1,2}$ \\ ${ }^{1}$ National Center for International Research of Biological Targeting Diagnosis and Therapy, \\ Guangxi Key Laboratory of Biological Targeting Diagnosis and Therapy Research, Collaborative Innovation \\ Center for Targeting Tumor Diagnosis and Therapy, Guangxi Medical University, Nanning, Guangxi 530021; \\ ${ }^{2}$ Department of Immunology, Guangxi Medical University, Nanning, Guangxi 530021, P.R. China
}

Received November 26, 2015; Accepted December 31, 2015

DOI: $10.3892 / o r .2016 .4866$

\begin{abstract}
Detection of leukemia at the early stage with high sensitivity is a significant clinical challenge for clinicians. In the present study, we developed a sensitive detector consisting of the product of oligonucleotides hybridized with semiconductor quantum dots (QDs) to generate a stronger fluorescent signal so that leukemic cells can be captured. In the present study, a biotin-modified Sgc8 aptamer was used to identify CCRF-CEM cells, and then biotin-appended QDs were labeled with the aptamer via streptavidin and biotin amplification interactions. We described the complex as QDs-bsb-apt. CEM and Ramos cells were used to assess the specificity and sensitivity of the novel complex. These results revealed that the complex could be more effective in diagnosing leukemia at the early stage. In conclusion, an innovative structure based on aptamer and QDs for leukemia diagnosis was provided. It has the potential to image tumor cells in vitro or in vivo and to realize the early diagnosis of disease. Furthermore, it may be used to provide guidance for clinicians to implement individualized patient therapy.
\end{abstract}

\section{Introduction}

It is well known that leukemia, bone marrow or blood cancer, is one of the most common and aggressive cancers (1),

Correspondence to: Professor Xiaoling Lu or Professor Yongxiang Zhao, National Center for International Research of Biological Targeting Diagnosis and Therapy, Guangxi Key Laboratory of Biological Targeting Diagnosis and Therapy Research, Collaborative Innovation Center for Targeting Tumor Diagnosis and Therapy, Guangxi Medical University, Nanning, Guangxi 530021, P.R. China E-mail: luwuliu@163.com

E-mail: yongxiang_zhao@126.com

*Contributed equally

Key words: aptamer, quantum dots, leukemia, tumor, toxicity, streptavidin, biotin particularly in children and adults (2). For conducting effective treatment of this disease, accurate and sensitive diagnoses are essential. In previous studies, DNA-silver nanocluster $(3,4)$, mass spectrometry (5) and aptamer-DNA concatamer-quantum dot probes (6) were used as a complex to detect leukemia cells. However, such complexes have serious drawbacks, such as insensitivity and high expense, and sophisticated instruments and meters are required. Hence, a simpler strategy with higher sensitivity is demanded to accurately detect leukemia cells.

Aptamers, special three-dimensional structures, can be obtained via SELEX (systematic evolution of ligands by exponential enrichment) in vitro process $(7,8)$. The simulated single-stranded oligonucleotides can selectively recognize as well as combine with a wide range of objects, such as drugs, proteins and even entire cells $(9,10)$. In comparison with conventional antibodies, aptamers show some superiority, including the capability of being synthesized easily with low expense, the ability to be purified to a high level, lack of immunogenicity and good stability (11-13), which are helpful to detect cancer cells $(14,15)$. The Sgc8 aptamer, selected based on the whole cell (16), has been proven to have characteristics including high binding-affinity and specificity for human T-acute lymphocytic leukemia (T-ALL) cell lines (17). CCRF-CEM, a T-ALL cell line, was reported to be used as a target cell, and Romas, a human Burkitt's lymphoma cell line was reported to be used as a negative control.

Fluorescent quantum dots (QDs) and nanometer semiconductors, have been used as photographic developers in the construction of diagnostic instruments and antitumor nanodrugs (18-21). They have attracted widespread attention due to their superior fluorescent features. Unlike precious metal nanoparticles and traditional organic dyes, QDs exhibit excellent biocompatibility, low toxicity, resistance to light bleaching, and allows stable and easy modulation (22). Several studies have confirmed that QDs are appropriate and efficient for PDT and bio-imaging (23-25). Furthermore, they can be applied to detect and sense biomarkers, molecular markers, and cell surface receptors when being linked with some guiding instruments or targeting molecules (26-28).

In the present study, we developed an innovative complex based on the Sgc8 aptamer and QDs for the diagnosis of 
leukemia. The biotin-modified Sgc8 aptamer was used to identify CCRF-CEM cells. Then biotin-appended QDs were labeled with the aptamer via streptavidin and biotin amplification interactions. The sensitive detection of leukemia cells by the complex (QDs-bsb-apt) was observed by quantifying the fluorescence signal of the QDs.

\section{Materials and methods}

Reagents. Streptavidin was purchased from Promega Corporation (Madison, WI, USA). Biotin-QD525 was synthesized by Beijing Zhongke Wu Yuan Biotechnology Co., Ltd. (Beijing, China) and the biotin-Sgc8 aptamer was synthesized by Shanghai Sangon Biotechnology (Shanghai, China). The sequence was: biotin- $\mathrm{T}_{10}, 5^{\prime}$-ATCTAACTGCTGCGCCGCCG GGAAAATACTGTACGGTTAGA-3'; and biotin-labeled random DNA library (biotin-Lib) as control biotin- ${ }_{10}, 5^{\prime}-\mathrm{NNN}$ NNNNNNNNNNNNNNNNNNNNNNNNNNNNNNNNN NNNNN-3'. Sterile phosphate-buffered saline (PBS; $10 \mathrm{mM}$, $\mathrm{pH}, 7.4)$ was used as the buffer. All solutions were prepared with Milli-Q water.

Cells and animals. All cells were provided by the National Center for International Research of Biological Targeting Diagnosis and Therapy, Guangxi Medical University (Nanning, Guangxi, China). CEM and Ramos cells were used as the positive and negative cells, respectively, for the QDs-bsb-Sgc8 complex. 293T cells were used to assess the toxicity of the complex. All of the cells were cultured in 1640 medium, which was supplemented with $1 \%$ penicillin-streptomycin and $10 \%$ fetal bovine serum (FBS) (both from Gibco Co., Grand Island, NY, USA). All of the cell lines were cultured at $37^{\circ} \mathrm{C}$, in a humid environment with $5 \% \mathrm{CO}_{2}$. Nude mice were provided by the Guangxi Laboratory Animal Center (Guangxi, China), and were raised and cared for following the Federation of European Laboratory Animal Science Association guidelines. All protocols were approved by the Animal Ethics Committee of Guangxi Medical University.

Preparation of the QD-aptamer complex. Biotin-QD525 was decorated by streptavidin, and then conjugated with the biotin-modified Sgc8 aptamer. Firstly, streptavidin and biotinQDs were mingled at a ratio of 10:1 and incubated for $40 \mathrm{~min}$ at $37^{\circ} \mathrm{C}$ to obtain streptavidin-QDs. Secondly, QDs-bsb-apt was synthesized followed by addition of the biotin-modified Sgc8 aptamers and incubated sequentially at a ratio of 40:1. Then, the mixture was co-cultured for $40 \mathrm{~min}$ at $37^{\circ} \mathrm{C}$. Finally, unconjugated biotin-aptamers were eliminated, and conjugated QDs-bsb-aptamer complexes were retrieved by centrifugal filtration at 14,000 rpm for $25 \mathrm{~min}$. Following washing in PBS (10 mM; pH, 7.4), an ultrasonic processor (Scientz-IID; Ningbo Scientz Biotechnology Co., Ltd., China) was used to disperse the aggregated conjugates by sonication ( $25 \mathrm{kHz}$; amplitude, $15 \mu \mathrm{m}$; sonication time, $3 \mathrm{~min}$ ) and stored at $4^{\circ} \mathrm{C}$.

Flow cytometric analysis. The cultured cells were collected and centrifuged for $8 \mathrm{~min}$ at 1,200 rpm at $4^{\circ} \mathrm{C}$, and washed two or three times in cold PBS. To determine the cell density, a counting plate was used. Then, $3-4 \times 10^{5}$ cells were resuspended in $200 \mu \mathrm{l}$ PBS. Cell samples were incubated with the QDs-bsb-apt complexes in cell culture medium or binding buffer $(200 \mu \mathrm{l})$ containing FBS [10\% (vol/vol)] on ice for $50 \mathrm{~min}$, followed by washing using washing buffer and suspension in serum $(200 \mu \mathrm{l})$. The detection sensitivity of this complex was determined by a flow cytometer (Epics XL; Beckman Coulter, USA). EXPO32 ADC analysis software was applied to analyze the data.

Cell imaging. CEM and Ramos cells were cultured in a 6-well cell culture plate at a density of $3-4 \times 10^{5}$ cells $/ \mathrm{ml}$. After incubation for $24 \mathrm{~h}$, the cells were then placed on ice for $30 \mathrm{~min}$ to avoid non-specific binding. The cells were washed with cold PBS three times, and then fixed with $4 \%$ polyoxymethylene (Sigma-Aldrich). After incubation with the complexes $(10 \mathrm{nM})$ in binding buffer $\left(1 \mathrm{mM} \mathrm{CaCl}_{2}, 1 \mathrm{mM} \mathrm{MgCl}_{2}, 1 \mathrm{mg} / \mathrm{ml}\right.$ bovine serum albumin and $10 \% \mathrm{FBS}$ ) on ice for $50 \mathrm{~min}$, the cells were then washed three times. Next, the cells were mounted onto microscope slides using 4',6-diamidino-2-phenylindole dihydrochloride (DAPI) (Life Co., USA) to perform imaging analysis via a fluorescence microscope (DS-Ri1; Nikon Corporation, Tokyo, Japan).

MTT assay. Evaluation of cell toxicity of the QDs-bsb-apt was carried out by the methylthiazol tetrazolium (MTT) assay. The process involved in the MTT assay is briefly described as follows. Firstly, $293 \mathrm{~T}$ cells $\left(2 \times 10^{4}\right.$ in $\left.100 \mu \mathrm{l}\right)$ were seeded into 96-well plates and cultured overnight in an incubator. Then, the cells were treated with the complexes $(10,20,30,50$ and $100 \mathrm{nM}$ ) or PBS as a control group for 24 or $48 \mathrm{~h}$. After that, $10 \mu \mathrm{l}$ of MTT $(5 \mathrm{mg} / \mathrm{ml})$ was added to each well and the cells were incubated for a further $3 \mathrm{~h}$ at room temperature in a lucifugal place. Before adding the ethanol/dimethyl sulfoxide (DMSO) into the well, the medium in the well was discarded. After $2 \mathrm{~min}$, the absorbance was tested at $\lambda=570 \mathrm{~nm}$ with an ELISA microplate reader (Thermo Scientific, USA).

Histological analysis. Evaluation of systemic toxicity was carried out by analysis of the histologic evidence from the heart, liver, kidney, spleen and lung. Six-week-old nude mice were administered an intravenous injection of QDs-bsb-apt $(15 \mathrm{mg} / \mathrm{kg}$ ) or PBS in the control experiments through the tail vein. Then, changes in the clinical behavior of the treated mice were monitored every day. Mice were sacrificed after being treated for 20 days, for histological examination. Tissue samples of the organs, which were preserved in $10 \%$ formaldehyde solution, were dehydrated, following a routine strategy, and then embedded in paraffin. The paraffin sections were deparaffinized and immersed in distilled water, following a routine strategy. Then, hematoxylin and eosin staining was carried out in the following steps: i) coloration with hematoxylin solution for 5-15 min; ii) separation of color with acid or ammonia solution for a few seconds, respectively; iii) washing with running water for half an hour; iv) dehydration in $70 \%$ and $90 \%$ alcohol for 10 min sequentially; v) eosin staining for 2-3 min; and vi) dehydration, clearing and mounting with neutral gums. The negative control group was implemented using the same steps as described above. Hematoxylin and eosin solutions were purchased from Beijing Solarbio Co., China. 


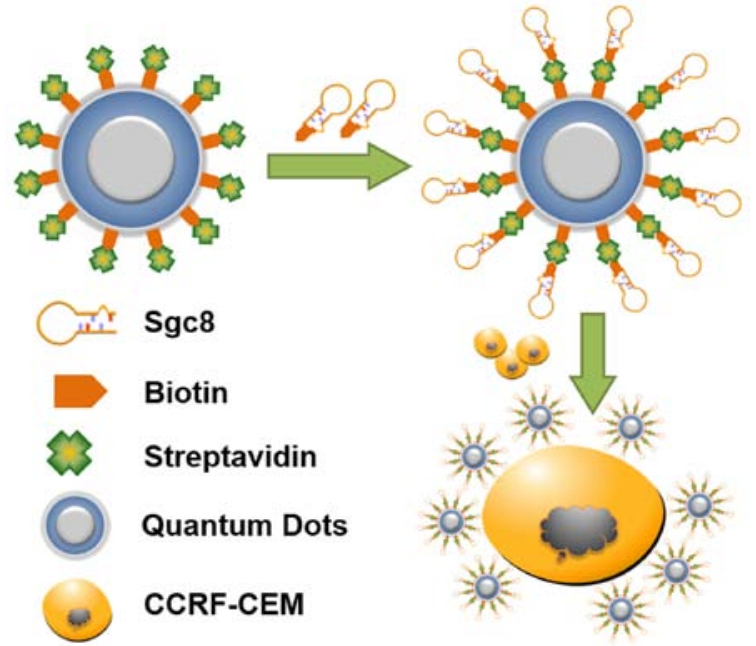

Figure 1. Illustration of the experimental principle: quantum dot complex based on aptamers which can be used for the specific targeting of leukemia cells.

Statistical analyses. Student's t-test was applied to make comparisons and one-way analysis of variance (ANOVA) was applied to compare three means or more. Data are shown as means \pm SD. All statistical analyses were two-tailed. $\mathrm{P} \leq 0.05$ was considered to indicate a statistically significant result. Statistical analyses were performed using GraphPad Prism software (GraphPad Software, San Diego, CA, USA).

\section{Results}

Experimental principle. Recently, QDs and aptamers have been combined and are widely used for cell imaging and biomedical application. In most cases, QD-aptamer conjugates are connected directly for cell recognition, such as biotin-QDs conjugated with a streptavidin-aptamer (29). Using such an approach, the aptamers in recognizing target cells may be baffled by steric hindrance caused by QDs, and the limited amplification may cause insensitive examination in detection processing. To avoid these issues, a strategy of linking biotinQDs and the biotin-aptamer was proposed in which biotin-QDs were coupled to streptavidin, and the streptavidin-appended QDs were then bound to the biotin-aptamer to allow the target cancer cells to be detected by fluorescence microscopy and flow cytometry (Fig. 1). CEM cells, a common leukemia cancer cell line, were treated as target cells. Sgc8, an aptamer of CEM cells, that has high affinity and specificity, was selected by cell-SELEX. This method may provide an efficient approach for tumor cell detection.

Characterization of the QDs-bsb-apt complex. Various characteristics of the prepared QDs-bsb-apt complex were analyzed via the following methods. Transmission electron microscopy (TEM; H-7650; Hitachi, Ltd., Tokyo, Japan) was employed to image the dissemination and the size. It was observed, via the image, that the QDs-bsb-apt complexes were well disseminated and the size was increased (Fig. 2A). The hydrodynamic diameter of the QDs-bsb-apt complexes was tested by dynamic light scattering. It was $18.92 \pm 3.05 \mathrm{~nm}$ (Fig. 2C).
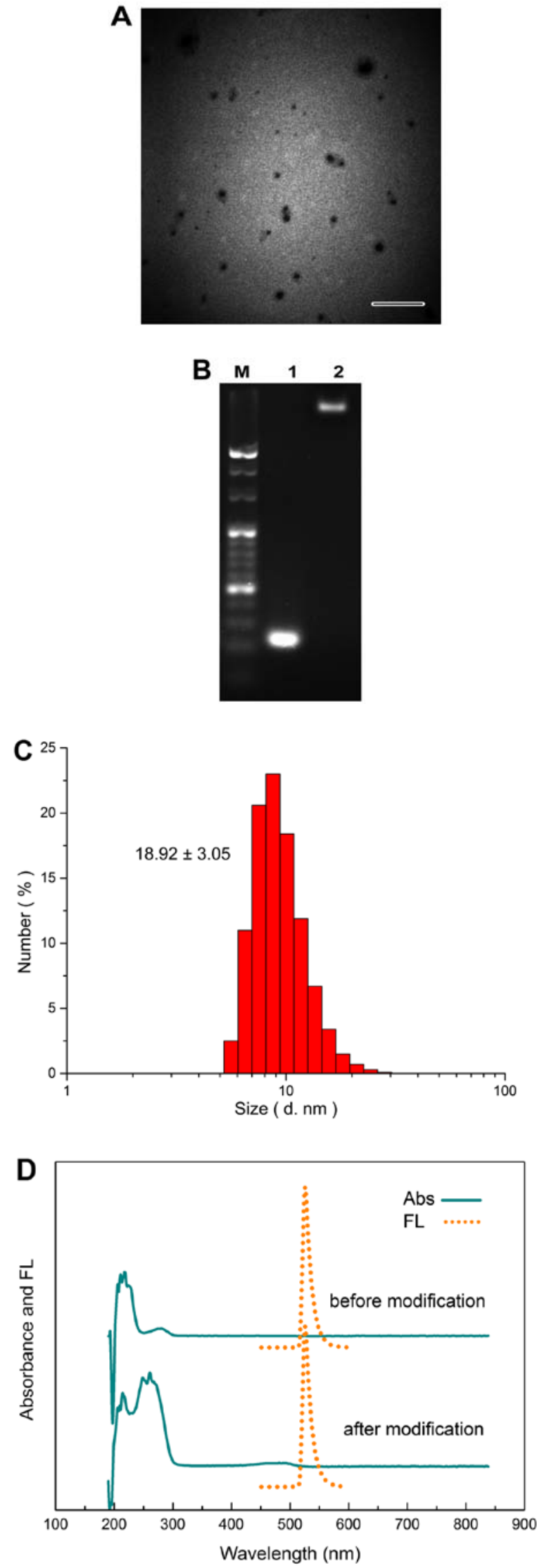

Figure 2. Characterization of the QDs-bsb-apt complex. (A) TEM image of the QDs-bsb-apt complex. Scale bar, $50 \mu \mathrm{m}$. (B) Gel electrophoresis free of QDs (lane 1) and QDs-bsb-apt (lane 2). The DNA size marker is presented in lane M. (C) Hydrodynamic diameter of the QDs-bsb-apt. (D) Absorbance (solid line) and fluorescence (dashed line) spectra of the aptamer or QDs before and after modification. 
A
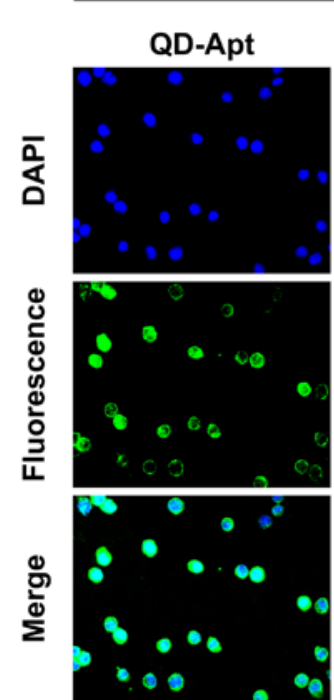

CCRF-CEM
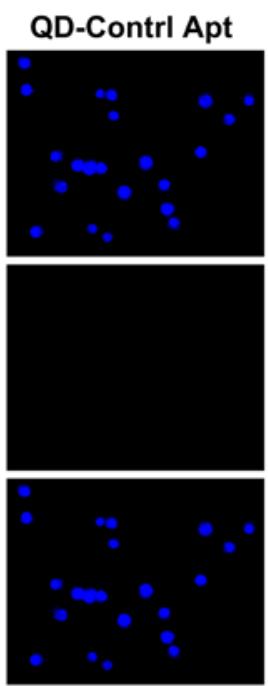

B

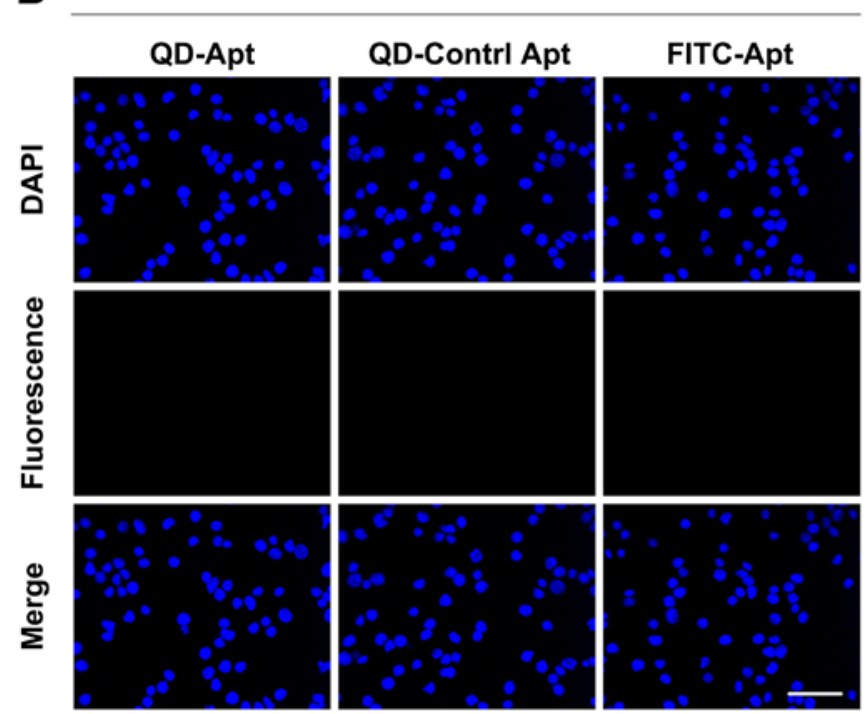

Figure 3. The specific detection of leukemia cells with the QDs-bsb-apt complex in buffer. (A and B) The top panels show fluorescent images of cell nuclei labeled with DAPI stain, the middle panels show fluorescent images of the complex and each of two control groups: QDs-bsb-Sgc8 (QD-Apt), QDs-bsb-Lib (QD-Contrl Apt) and FITC-Sgc8 (FITC-Apt), and the bottom panels show the merger of DAPI and fluorescence images of the complex and each of the two control groups. CCRF-CEM (A) and Ramos (B) cells were incubated with QD-Apt, QD-Contrl Apt or FITC-Apt. Scale bars, $50 \mu \mathrm{m}$ (fluorescence microscopy at a magnification of $\mathrm{x} 400)$.

A

CCRF-CEM
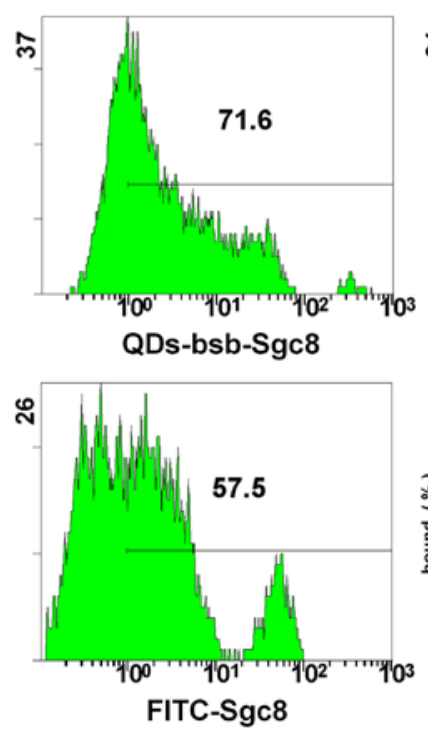

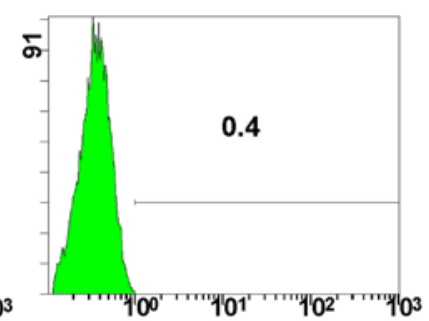

QDs-bsb-Lib

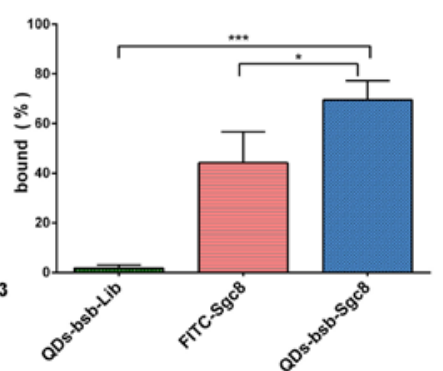

B

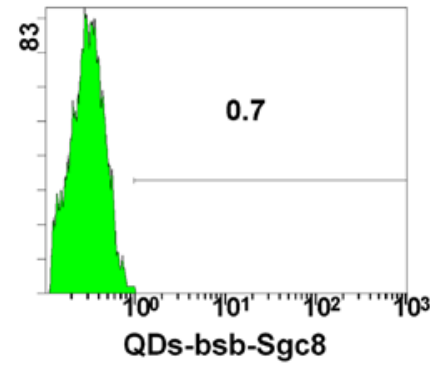

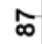

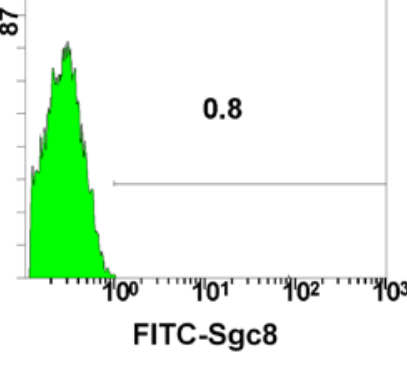

Ramos
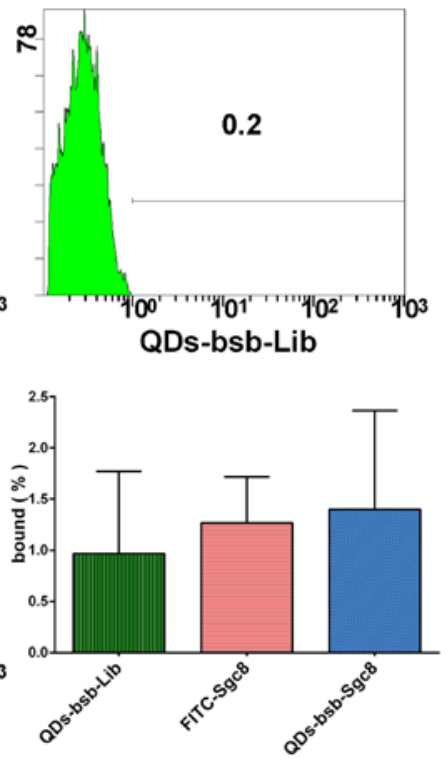

Figure 4. Sensitive detection of leukemia cells using the QDs-bsb-apt complex in serum. (A) CCRF-CEM cells were incubated with QDs-bsb-Sgc8, QDs-bsb-Lib or FITC-Sgc8 in serum, respectively. (B) Ramos were incubated with QDs-bsb-Sgc8, QDs-bsb-Lib or FITC-Sgc8 in serum, respectively, as well.

The fluorescence emission peak of the QDs with or without the aptamer was $525 \mathrm{~nm}$ and the absorbance peak of the aptamers with or without QDs was $260 \mathrm{~nm}$ (Fig. 2D). Electrophoretic mobility shift assay was performed using $3 \%$ agarose gel electrophoresis. As shown in Fig. 2B, the band in lane 2 indicates the QDs-bsb-apt complex. Its size is $>500 \mathrm{bp}$. The band in lane 1 represents the aptamer alone. Its size is $\sim 50 \mathrm{bp}$ ).

Fluorescence microscopic analysis demonstrates the high sensitivity of the detection of leukemia cells using
$Q D s$-bsb-apt in buffer. At first, feasibility analysis of the QDs-bsb-apt strategy was carried out using fluorescence microscopy. Human Burkitt's lymphoma Romas cells were used as negative control and the random DNA library (lib) was used as a control sequence. In the fluorescence images, CEM cells exerted a distinctly stronger fluorescence intensity on their surface with the presence of QDs-bsb-Sgc8 than that of FITC-Sgc8, while no fluorescence signal was observed for the random library (Fig. 3A). When CCRF-CEM cells were replaced by Ramos cells, no obvious fluorescence signal 


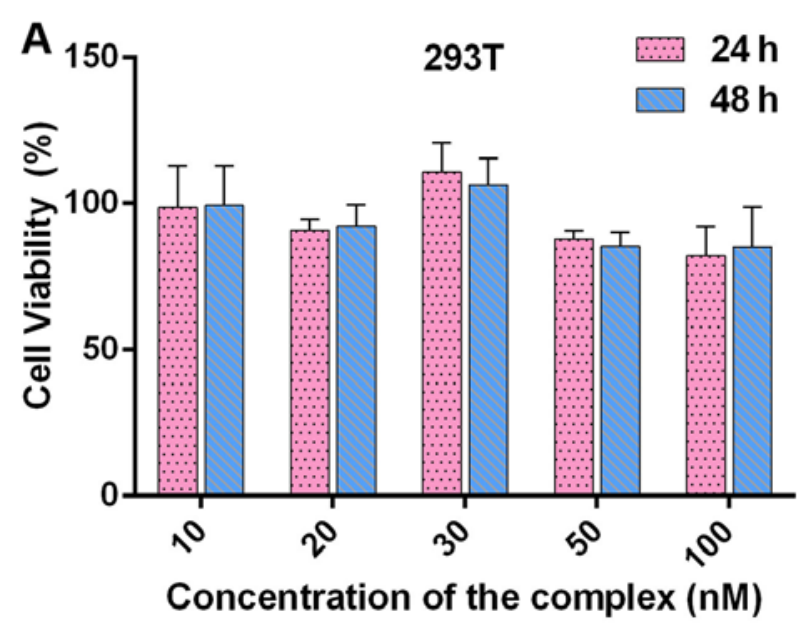

B

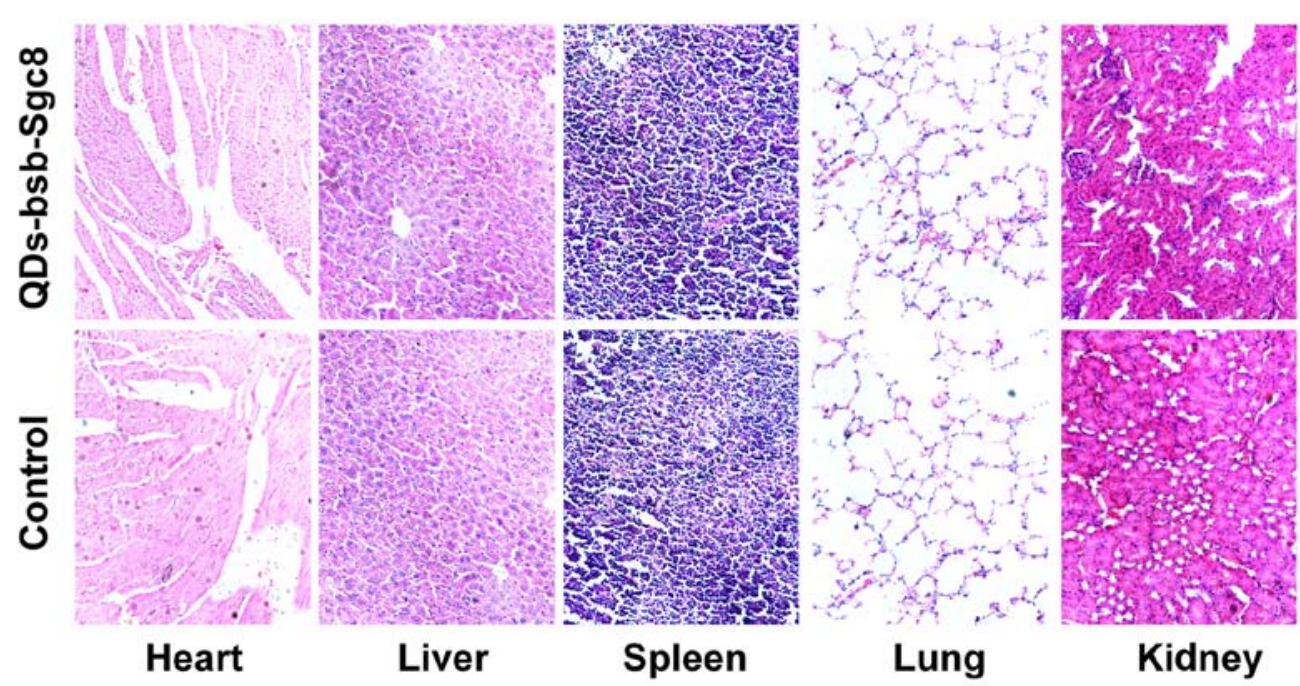

Figure 5. Non-toxicity of the QDs-bsb-apt complex to 293T normal cells (A) and organs such as the heart, liver, spleen, lung and kidney (B). 293T cells were incubated with various concentrations of the QDs-bsb-Sgc8 complex (10, 20,30, 50 and $100 \mathrm{nM}$ ) for $24 \mathrm{~h}$ (red column) or $48 \mathrm{~h}$ (blue column). (B) Comparative safety assessment of QDs-bsb-Sgc8 compared with the PBS group 20 days following a single intravenous administration in nude mice (n=3). No histologic evidence of toxicity in any organ was identified.

was observed (Fig. 3B). This result indicated that due to the the specific properties of the Sgc8 aptamer, QDs-bsb-Sgc8 complexes were able to be applied in the detection of the CEM cells with high sensitivity.

Flow cytometric analysis demonstrates the high sensitivity of detection of leukemia cells using QDs-bsb-apt in serum. Flow cytometric analysis confirmed that the positive CEM cells shown a stronger fluorescent signal property in serum than the QDs-bsb-Lib, Ramos cells and the FITC-Sgc8 (Fig. 4). CEM cells $(71.6 \%)$ could be detected by employing the QDs-bsb-Sgc8 complex, while $57.5 \%$ of cells were detected by FITC-Sgc8. The detection efficiency of QDs-bsb-Sgc8 was 1.2-fold higher than the traditional organic dye modified aptamer FITC (Fig. 4).

The QDs-bsb-apt complex exhibits non-toxicity. MTT assay demonstrated that various concentrations of the QDs-bsb-apt complexes were non-toxic to normal cells such as $293 \mathrm{~T}$ when co-incubated with the QDs-bsb-apt complexes (Fig. 5A).
Additionally, after injecting the QDs-bsb-apt complexes into nude mice, hematoxylin and eosin staining was conducted. The staining results demonstrated that the complexes were non-toxic to the entire organism (Fig. 5B).

\section{Discussion}

The synthesis of an aptamer with quantum dots (QDs) in this strategy is a simple and efficient method for generating a complex to detect tumor cells. Such a complex possesses the characteristics of high and specific affinity, low immunogenicity, high photoluminescence quantum yield and a remarkable high extinction ratio. The synthesis of the Sgc8 aptamer with QDs creates a hybrid complex possessing properties from both the aptamer and the QDs. The Sgc8 aptamer provides specific binding to CEM cells, while the QDs supply a sensitive signal for accurate detection.

To demonstrate that the QDs-bsb-apt complex was synthesized successfully, various properties were assessed. TEM imaging and hydrodynamic diameter detection showed that the 
complexes were satisfactory. The increased size and its narrow distribution suggested that the complexes were uniform and well-proportioned. Fluorescence emission spectra and absorbance of the nucleic acid showed that biotin-QDs modified by the biotin-aptamer preserved the characteristics of the aptamers and the superior spectral properties of the QDs after modification. To further determine the formation of the QDs-bsb-apt complex, electrophoretic mobility shift assay was performed using agarose gel electrophoresis. QDs-bsb-apt was hardly run on the gel, which indicated that the complex was synthesized successfully and unconjugated aptamers were removed.

To illustrate the sensitive detection of leukemia cells using the QDs-bsb-Sgc8 complex, fluorescence microscopy and flow cytometric analysis were carried out. The fluorescent images demonstrated that the CEM cells incubated with QDs-bsb-Sgc8 exhibited stronger fluorescent intensity than that with FITC-Sgc8, which indicated that the QDs-bsb-apt complex had the ability to target cancer cells specifically. Furthermore, the efficiency of detection of the complex was higher than that of the FITC-apt. In the flow cytometric analysis, we observed some shift in the fluorescence intensity among the positive and control samples, suggesting that the sensitivity of this complex was higher. We presumed that the reason for this was that our method of synthesis of the QDs-bsb-apt complex facilitated the binding of more aptamers with QDs to increase the specificity and fluorescence intensity. On the basis of the above results of the fluorescence imaging and the flow cytometric analysis, we conclude that the sensitivity of the QDs-bsb-apt complex was better than that of the FITC-apt complex.

To confirm the non-toxicity of the QDs-bsb-apt complex, MTT assay and hematoxylin and eosin staining of histological sections were executed. The results showed that the complex was non-toxic to normal cells and to the whole organism, which suggests that the complex could not only be safely used as a detector in vitro, but also has the potential to be used safely in vivo for tumor assessment or treatment. After referring to the reported literature, it was quite meaningful that the present study described the toxicity of the QDs-bsb-apt complex for the first time, which could lay a foundation for further research on the aptamer-conjugated QD complex.

In the previous study, the Sgc8 aptamer selected by Shangguan et al (16) was combined with organic dyes or metal elements. For instance, aptamer-silver conjugates were used as theragnostic agents for specific cancer cell therapy and fluorescence-enhanced cell imaging by Li et al (3). In addition, aptamer-DNA concatamer-quantum dots probes were used for selective and ultrasensitive detection of cancer cells by Liu et al (6), and a DNA-silver nanocluster fluorescence was applied in cancer cell detection by Yin et al (4). These conjugates were applied to capture leukemia cells for detection or cure. The present study extends the previous research: i) by demonstrating that QDs conjugated with the Sgc8 aptamer can detect CEM cells efficiently; ii) by demonstrating that the method of conjugating biotin-QDs with biotin-Sgc8 aptamer through coupling of biotin-QDs to streptavidin is more sensitive than the FITC-labeled aptamer directly; and iii) by proving the non-toxicity of the QDs-bsb-apt complex at the cell and systemic level.

In summary, an innovative structure based on aptamer and QDs for leukemia diagnosis was provided. We showed that QDs-bsb-Sgc8 can recognize CEM cells specifically and sensitively. This complex exhibits properties of both the aptamer and QDs, including specificity of targeting and sensitivity to fluoresce. The connections to chemistry also enable it to be used for tumor cell imaging in vitro or in vivo, for clinical application to realize the diagnosis of disease at the early stage of the development of tumors. Furthermore, future investigations may focus on constructing a drug transporter combined with such a complex, which could be used for simultaneously treating and detecting tumors without toxicity and with high specificity. In this way, clinicians may be provided with significant and timely guidance through the detection signal to implement individualized patient therapy. Our research group will conduct further research.

\section{Acknowledgements}

The present study was supported, in part, by grants from the National Natural Scientific Foundation of China (nos. 81430055 and 81372452), Programs for Changjiang Scholars and Innovative Research Team in University (No.IRT_15R13), the International Cooperation Project of the Ministry of Science and Technology of China (no. 20 15DFA31320), the Project for Innovative Research Team in Guangxi Natural Science Foundation (2015GXNSFFA139001), and the Project of Science and Technology of Guangxi (nos. 14125008-2-12 and 1599005-2-10).

\section{References}

1. Nelson BP, Treaba D, Goolsby C, Williams S, Dewald G, Gordon L and Peterson LC: Surface immunoglobulin positive lymphoblastic leukemia in adults; A genetic spectrum. Leuk Lymphoma 47: 1352-1359, 2006.

2. Karanu FN, Murdoch B, Miyabayashi T, Ohno M, Koremoto M, Gallacher L, Wu D, Itoh A, Sakano S and Bhatia M: Human homologues of Delta-1 and Delta- 4 function as mitogenic regulators of primitive human hematopoietic cells. Blood 97: 1960-1967, 2001

3. Li H, Hu H, Zhao Y, Chen X, Li W, Qiang W and Xu D: Multifunctional aptamer-silver conjugates as theragnostic agents for specific cancer cell therapy and fluorescence-enhanced cell imaging. Anal Chem 87: 3736-3745, 2015.

4. Yin J, He X, Wang K, Xu F, Shangguan J, He D and Shi H: Label-free and turn-on aptamer strategy for cancer cells detection based on a DNA-silver nanocluster fluorescence upon recognition-induced hybridization. Anal Chem 85: 12011-12019, 2013.

5. Xiong L, Zhang J, Yuan B, Dong X, Jiang X and Wang Y: Global proteome quantification for discovering imatinib-induced perturbation of multiple biological pathways in K562 human chronic myeloid leukemia cells. J Proteome Res 9: 6007-6015, 2010.

6. Liu H, Xu S, He Z, Deng A and Zhu JJ: Supersandwich cytosensor for selective and ultrasensitive detection of cancer cells using aptamer-DNA concatamer-quantum dots probes. Anal Chem 85: 3385-3392, 2013.

7. Ellington AD and Szostak JW: In vitro selection of RNA molecules that bind specific ligands. Nature 346: 818-822, 1990.

8. Tuerk C and Gold L: Systematic evolution of ligands by exponential enrichment: RNA ligands to bacteriophage T4 DNA polymerase. Science 249: 505-510, 1990.

9. Tan W, Donovan MJ and Jiang J: Aptamers from cell-based selection for bioanalytical applications. Chem Rev 113: 2842-2862, 2013

10. Chen H, Yuan F, Wang S, Xu J, Zhang Y and Wang L: Aptamer-based sensing for thrombin in red region via fluorescence resonant energy transfer between $\mathrm{NaYF}_{4}: \mathrm{Yb}, \mathrm{Er}$ upconversion nanoparticles and gold nanorods. Biosens Bioelectron 48: 19-25, 2013. 
11. Vinkenborg JL, Mayer G and Famulok M: Aptamer-based affinity labeling of proteins. Angew Chem Int Ed Engl 51: 9176-9180, 2012

12. Xiao Z and Farokhzad OC: Aptamer-functionalized nanoparticles for medical applications: Challenges and opportunities. ACS Nano 6: 3670-3676, 2012.

13. Gong Q, Wang J, Ahmad KM, Csordas AT, Zhou J, Nie J, Stewart R, Thomson JA, Rossi JJ and Soh HT: Selection strategy to generate aptamer pairs that bind to distinct sites on protein targets. Anal Chem 84: 5365-5371, 2012.

14. Famulok M, Hartig JS and Mayer G: Functional aptamers and aptazymes in biotechnology, diagnostics, and therapy. Chem Rev 107: 3715-3743, 2007.

15. Liang H, Zhang XB, Lv Y, Gong L, Wang R, Zhu X, Yang R and Tan W: Functional DNA-containing nanomaterials: Cellular applications in biosensing, imaging, and targeted therapy. Acc Chem Res 47: 1891-1901, 2014.

16. Shangguan D, Li Y, Tang Z, Cao ZC, Chen HW, Mallikaratchy P, Sefah K, Yang CJ and Tan W: Aptamers evolved from live cells as effective molecular probes for cancer study. Proc Natl Acad Sci USA 103: 11838-11843, 2006.

17. Fang $X$ and Tan W: Aptamers generated from cell-SELEX for molecular medicine: A chemical biology approach. Acc Chem Res 43: 48-57, 2010.

18. Zhang M, Liu H, Chen L, Yan M, Ge L, Ge S and Yu J: A disposable electrochemiluminescence device for ultrasensitive monitoring of K562 leukemia cells based on aptamers and $\mathrm{ZnO@carbon} \mathrm{quantum} \mathrm{dots.} \mathrm{Biosens} \mathrm{Bioelectron} \mathrm{49:} \mathrm{79-85,}$ 2013.

19. Wang FB, Rong Y, Fang M, Yuan JP, Peng CW, Liu SP and Li Y: Recognition and capture of metastatic hepatocellular carcinoma cells using aptamer-conjugated quantum dots and magnetic particles. Biomaterials 34: 3816-3827, 2013.

20. Sheng Z, Hu D, Zhang P, Gong P, Gao D, Liu S and Cai L: Cation exchange in aptamer-conjugated CdSe nanoclusters: A novel fluorescence signal amplification for cancer cell detection. Chem Commun 48: 4202-4204, 2012.
21. Hu D, Zhang P, Gong P, Lian S, Lu Y, Gao D and Cai L: A fast synthesis of near-infrared emitting CdTe/CdSe quantum dots with small hydrodynamic diameter for in vivo imaging probes. Nanoscale 3: 4724-4732, 2011.

22. Medintz IL, Uyeda HT, Goldman ER and Mattoussi H: Quantum dot bioconjugates for imaging, labelling and sensing. Nat Mater 4: 435-446, 2005.

23. Lee J, Kang HJ, Jang H, Lee YJ, Lee YS, Ali BA, Al-Khedhairy AA and Kim S: Simultaneous imaging of two different cancer biomarkers using aptamer-conjugated quantum dots. Sens Basel 15: 8595-8604, 2015.

24. Alibolandi M, Abnous K, Ramezani M, Hosseinkhani $\mathrm{H}$ and Hadizadeh F: Synthesis of AS1411-aptamer-conjugated CdTe quantum dots with high fluorescence strength for probe labeling tumor cells. J Fluoresc 24: 1519-1529, 2014.

25. Mashinchian O, Johari-Ahar M, Ghaemi B, Rashidi M, Barar J and Omidi Y: Impacts of quantum dots in molecular detection and bioimaging of cancer. Bioimpacts 4: 149-166, 2014.

26. Xing $\mathrm{Y}$ and Rao J: Quantum dot bioconjugates for in vitro diagnostics \& in vivo imaging. Cancer biomarkers: Section. Dis Markers 4: 307-319, 2008

27. Savla R, Taratula O, Garbuzenko O and Minko T: Tumor targeted quantum dot-mucin 1 aptamer-doxorubicin conjugate for imaging and treatment of cancer. J Control Release 153: 16-22, 2011.

28. Tada $\mathrm{H}$, Higuchi $\mathrm{H}$, Wanatabe TM and Ohuchi N: In vivo real-time tracking of single quantum dots conjugated with monoclonal anti-HER2 antibody in tumors of mice. Cancer Res 67: $1138-1144,2007$.

29. Wu C, Liu J, Zhang P, Li J, Ji H, Yang X and Wang K: A recognition-before-labeling strategy for sensitive detection of lung cancer cells with a quantum dot-aptamer complex. Analyst 140: 6100-6107, 2015 